\title{
Enhancing the Interoperability of Multimedia Learning Objects Based on the Ontology Mapping
}

\author{
http://dx.doi.org/10.3991/ijet.v9i5.3934 \\ Jihad Chaker, Souhaib Aammou and Mohamed Khaldi \\ Université Abdelmalek Essaadi, Tétouan, Maroc
}

\begin{abstract}
This article addresses the interoperability between the semantic learning platforms and the educational resources banks, more precisely between the LOM and MPEG-7 standards. LOM is a set of metadata associated with e-learning content, while MPEG-7 is a standard for describing multimedia content. The use of educational resources has become an essential component to meet the learning needs. Given the multimedia nature of these resources, such use causes problems in the interoperability of multimedia learning objects in e-Learning environments, indexing and retrieval of digital resources. Faced with these problems, we propose a new approach for the multimedia learning objects by using the ontology mapping between the LOM and MPEG-7 ontologies.
\end{abstract}

Index Terms-LOM, MPEG-7, Ontology mapping, Local similarity.

\section{INTRODUCTION}

With the observed increase in the use of multimedia content in the e-Learning environments, the specialists in the field of engineering Educational Multimedia, consider the support of multimedia by the e-learning systems as an essential criterion for enhance the learning process, making it more natural and spontaneous operation. And we value the non-linear thinking by giving more freedom to the learner to determine its own way of learning.

The need for digital resources is a intournable action in so far as it gives more freedom to learners to personalize their learning path, in fact, to achieve this goal, several organizations have set up a banks of digital resources.

The mass and nature of digital resources involve the use of a standard to facilitate the search and indexing of these resources, the standard MPEG-7 is, in our approach, a framework to create the metadata of multimedia learning objects .But the nature of the multimedia content does not favor the semantics, which reflects negatively at the main characteristics of learning objects such as reuse, flexibility, adaptability and interoperability.

We discuss in this article, the multimedia content description, then the solution mapping ontologies based on the correspondence between the two ontologies source.

\section{Multimedia CONTENT DESCRIPTION INTERFACE USING MPEG-7}

\section{A. Metadata, educational metadata}

The concept of learning object has a rich terminology such as learning resources, digital learning object...
According to the group LTSC (Learning Technology Standards Committee) of the IEEE (Institute of Electrical and Electronics Engineers), a learning object is defined as «Learning Objects are defined here as any entity, digital or non-digital, which can be used, re-used or referenced during technology supported learning» [1]. While the concept of learning object can be: tutor, website, student, document, instructional sequence, simulation, video, school. In several research projects, can be seen that the concept of learning object covers more non-numeric entities, in this sense, Strijker defines learning objects « as digital entities, available for use or reuse in different learning settings» [2].

The definition of medata, differs depending on the area covered, literally metadata is data representing other data, according to Greenberg « as structured data about an object that supports functions associated with the designated object» [3]. In an educational context, metadata is used to facilitate research and the use of learning objects. . Metadata allows systems, applications and users to manage and access resources without a need for interaction with the resource itself [4].

According to ISO ( the International Organisation for Standardisation) :"Standards are documented agreements containing technical specifications or other precise criteria to be used consistently as rules, guidelines, or definitions of characteristics to ensure that materials, products, processes, and services are fit for their purpose". As part of the development of e-learning, several standards have been created to guide its practices. Thus, the content used generally meet one or more of the standard, and following the consensus of organizational actors of the educational world, four major characteristics of standard e-earning should have the following characteristics: Accessibility, Interoperability, reusability and Sustainability. among which are:

- AICC (Aviation Industry CBT (Computer-Based Training) Committee): This standard defines the interoperability between learning platform and content of training. AICC is the result of an international association of airlines combining professional training.

- LOM (Learning Object Metadata) is an XML schema description defined by the IEEE. This schema is associated with a set of e-learning content metadata. These metadata are grouped into 9 categories.

- IMS (Instructional Management Systems) is a global consortium open to members of education, businesses, universities and governments for normalizing and 
describe a standard for knowledge objects and elearning content. Among the projects of the IMS are: Content Packaging, Question and Test Interoperablility, Learning Object Metadata, Learner Information Packaging, Enterprise Interoperability ...

- SCORM (Sharable Content Object Reference) is a set of technical specifications defined by the ADL (Advanced Distributed Learning) association to standardize the indexing and sharing educational content used in e-learning. This model includes himself several standards (AICC, LOM, IMS ...). SCORM standards allow the creation of grains course, reusable and interoperable.

\section{B. MPEG-7 Overview}

MPEG-7, formally named "Multimedia Content Description Interface", is a standard for describing the multimedia content data that supports some degree of interpretation of the information meaning, which can be passed onto, or accessed by, a device or a computer code. MPEG7 is not aimed at any one application in particular; rather, the elements that MPEG-7 standardizes support as broad a range of applications as possible [5].

MPEG-7 is a standard ISO and IEC (the International Electrotechnical Commission) for description of multimedia content and how objects are grouped into scenes. More precisely, the MPEG-7 in reality it is "bits about the bits." $\mathrm{He}$ is a member of the MPEG family "Moving Picture Experts Group." It is based on the combination of each description encoded in XML to specific content. this content differs depending on the context, it may be all or a portion of a video, a 3D model, an image or a sound.

MPEG-1, 2, 4 is to encode content and representation, however, the MPEG-7 is independent of the encoding technique or storing the contents of the document, it is used to build metadata (Content Description). This indicates that it is possible to establish a description of an MPEG-7 MPEG-1, MPEG-2 or MPEG-4, but you can do the same with a paper or an analog picture.

MPEG-7 aims to make multimedia content accessible recoverable filterable manageable, enable fast and efficient retrieval of information. MPEG-7 aims to: make multimedia content accessible recoverable filterable manageable. To enable fast and efficient retrieval of information. From time to time the application of this standard fields increase as media selection Broadcast, Digital libraries, E-Commerce, Education, Multimedia directory services, monitoring and remote sensing.

The objective is to define the minimum to ensure a certain degree of syntactic and semantic interoperability tools description. The description generation comport feature extraction, indexing process, annotation and it is not normative in the context of MPEG-7, the same for the consumption regarding the search engine, filtering tool, retrieval process and any other program that can use the description.

Figure 1 shows a highly abstract block diagram of a possible MPEG 7 processing chain, included here to explain the scope of the MPEG-7 standard.

Those main elements of the MPEG-7's standard are:

- Descriptors (D) representations of features, that 'define the syntax and the semantics of Each feature representation.

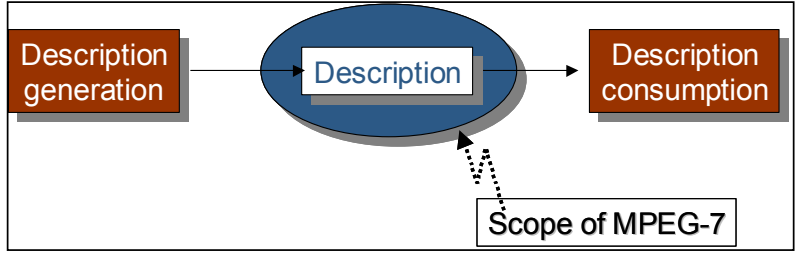

Figure 1. Scope of MPEG-7

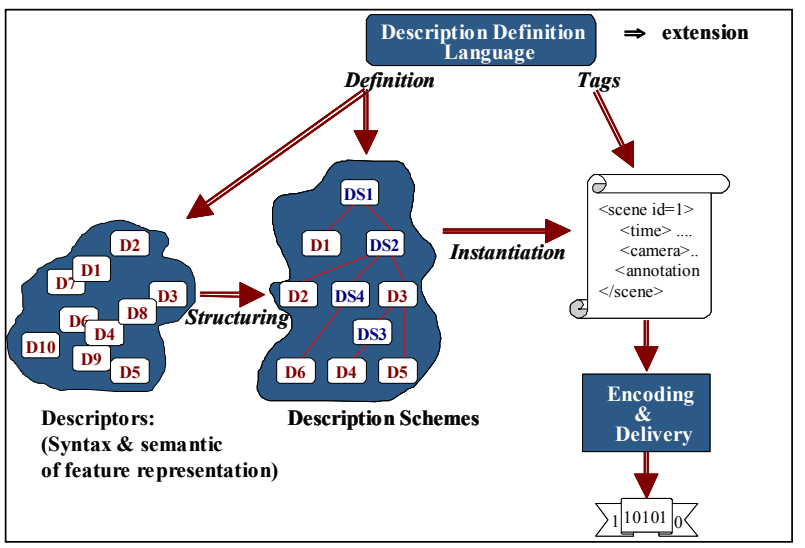

Figure 2. MPEG-7 main elements

- Description Schemes (DS) They specify the structure and semantic relations between their components, which can be either descriptors or descriptors schemes.

- Description Definition Language (DDL), It is based on XML, it is to define the syntax of the MPEG-7 Description Tools for. It also allows the creation of new DSs (and Possibly Ds), the extension and modification of DSs.

- System tools to carrier multiplexing of descriptions, synchronization issues, transmission Mechanisms, coded representations for efficient storage and transmission, management and protection of intellectual property in MPEG-7 descriptions.

Figure 2 shows the relationship among the different MPEG-7 elements introduced above.

The MDS (MPEG-7 Multimedia Description Schemes) refer to all kinds of media consisting of audio, visual and textual data, whereas the domain-specific descriptors, such as those for color, texture, shape, melody and so forth, refer specifically to the audio or visual domain [6]. The MDS metadata structures are expressed on the XML Schema, The MDS contains MPEG-7 Descriptors or other DSs.

The MDS is based on the content management and description schemes. The content management descriptors includes the life cycle of multimedia content, from its creation to its use, including information in the media to describe the storage format, the quality of media, media location.

\section{MPEG-7 Ontology}

Among the possible knowledge representations multimedia field,we found the services provided by the semantic web, and more specifically the ontologies that have a number of advantages, in terms of formal and explicit representation of conceptualizations. In this context, a number of ontologies media were based on the specific 
multimedia standard MPEG-7. Include: Harmony aceMedia, SmartWeb, Boemie, Rhizomik, DS-MIRF, COMM ...

This work is based on ontology Harmony [7], which is the result of an international collaboration funded by several organizations to represent the complex structural and semantic relations on multimedia resources. The formalization of components MPEG-7 MDS (more Audio and Visual parts) was presented with the RDFS language. The ontology developed was increased later and finally to DAML OWL illustrated in Figure 3.

\section{MAPPING}

\section{A. definition}

An ontology mapping can be defined formally as a pair $\mathrm{O}=(\mathrm{S}, \mathrm{A})$, where $\mathrm{S}$ is the ontology signature which describes the vocabulary, and $\mathrm{A}$ is a set of ontological axioms, these specify the intended interpretation of a particular domain. The ontological signature is modeled by a mathematical structure. For example, it could be a hierarchy of concept or class symbols modeled as a partial ordered set (poset), or a set of relations symbols whose arguments are defined over the concepts of the concept hierarchy [8].

In our case, multiple ontologies need to be accessed from several applications. Mapping could provide a common layer from which several ontologies could be accessed and hence could exchange information. A mapping can be seen as a collection of mapping rules all oriented in the same direction., from one ontology to the other, and such that the elements of the source ontology appear at most once. To find the correspondence between the ontologies we proceed by calculating the local similarity.

\section{B. Calculation of local similarity}

The calculation of the local similarity is carried out only once for each couple of nodes. The measurement of local similarity of the couples of entities is calculated via algorithm (function SIMTERM). The calculation of the similarity local (or terminological) is carried out between the descriptors of entities like the names, the comments, etc. The terminological similarity is made up of the syntactic similarity and the lexical similarity. Thus, the syntactic similarity is calculated via the functions of LEVEINSTEIN or EditDistance [9]. While the API of WORDNET [10] is exploited for calculation of lexical similarity. Function SIMTERM makes it possible to calculate the terminological similarities of the couples of nodes of two ontologies. It takes in entry two ontologies $\mathrm{O} 1$ and $\mathrm{O} 2$ to be aligned, represented in the shape of two OWLGraph graphs, as well as the function of terminological similarity to use and gives in return a vector of terminological similarity of each couple of nodes. The function CalculSimTerm (Algorithm, line 12) takes in entry two nodes $\mathrm{N} 1$ and N2, and turns over a value of similarity. This function is provided by one of the methods of calculating of following similarity: the measurement of LEVENSHTEIN, the distance from the under-chains or the API of WORDNET. The local similarity for the various couples of entities is exploited thereafter for the calculation of the total similarity.

\section{Experimental evaluation}

Experimental evaluation was conducted on the aspect of "intra-method" will focus on evaluating performance, ie, execution time, method vs. the change in the size of the ontologies to align, and the similarity measure used.

In what follows, we will try to measure the evolution of the performances of our method compared to the increase of the composition structural of ontology. Each test brings an incremental aspect of the composition structural of ontology. The tests carried out are three types of tests.

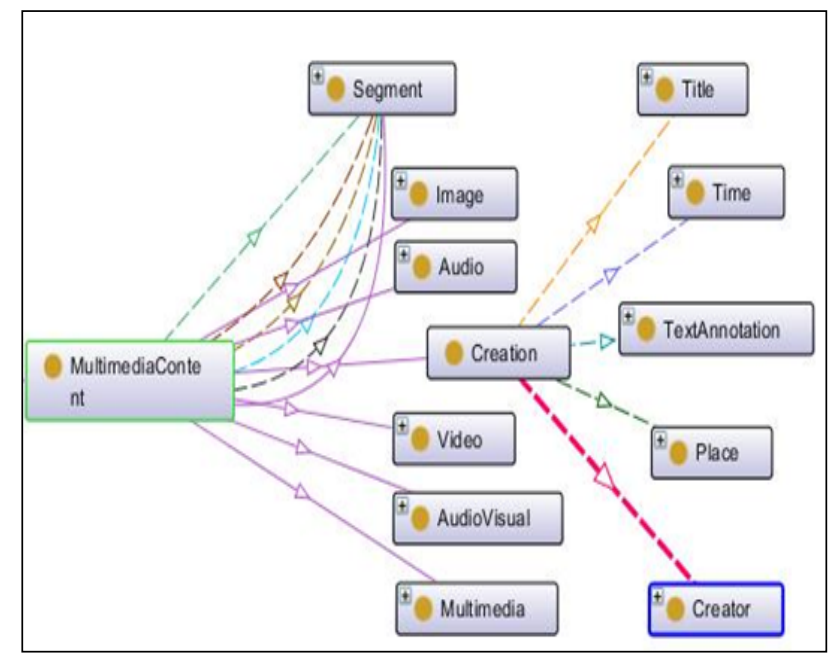

Figure 3. MPEG-7 ontology graph in protégé editor

- O1, O2: two ontologies (LOM and MPEG-7) to be mapped.

- VST: terminological vector of similarity

Each node of ontology presents among its characteristics the field:

- type: the type of the node

Each element of the vectors VST is characterized by the following fields:

- $\quad \mathrm{N} 1$ : the node of ontology O1

- $\mathrm{N} 2$ : the node of ontology O2

- Rhe value of similarity

Figure 4. Notations used in algorithm SIMTERM

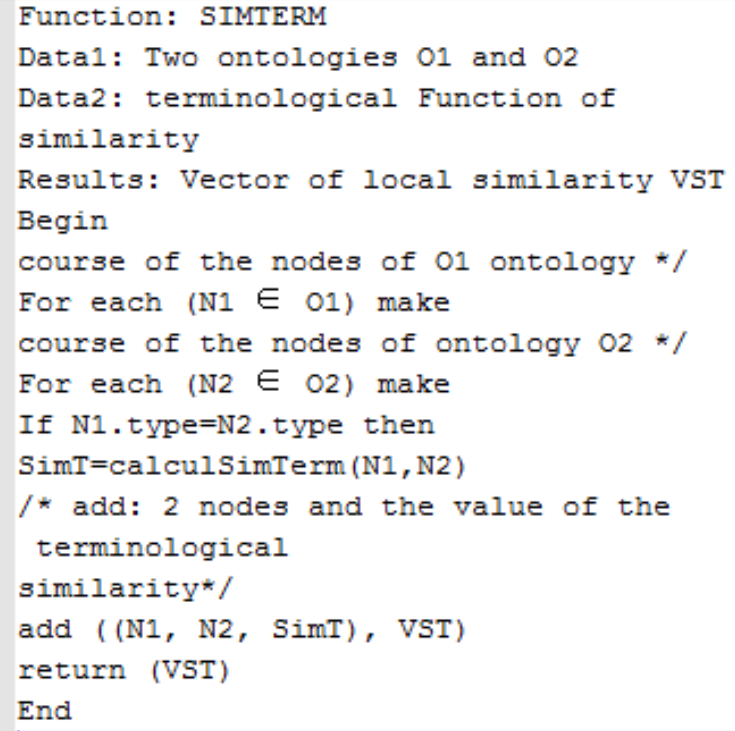

Figure 5. Algorithm SIMTERM 
TABLE I.

EXECUTION TIME OF OWL-GRAPH CONSTRUCTION AND SIMILARITY METHOD IN SECONDS.

\begin{tabular}{|l|c|c|c|}
\hline & TEST1 & TEST2 & TEST3 \\
\hline TE $:$ Construction OWL Graph & 1,700 & 2,300 & 3,340 \\
\hline TE $:$ LEVENSHTEIN & 60,235 & 111,234 & 165,230 \\
\hline TE $:$ WORDNET & 75,141 & 151,878 & 230,643 \\
\hline
\end{tabular}

In the TEST1, the ontology of reference is only made up of classes.. In the TEST2, the properties of nature object are added to the classes. In the TEST3, complete ontology is used,. According to the results presented in table 1, the performances depend on the two following aspects: size of ontologies and the choice of the function of terminological similarity. Indeed, the time of execution increases considerably when the number of entities increases and conversely.

The choice of the terminological function of similarity also influences over the execution tim. Indeed, the use of a simple function, like that of LEVENSHTEIN, for the calculation of the terminological similarity reduced the time execution. On the other hand, the use of a function more complex as the WORDNET increases considerably the execution time of the process of mapping. This variation is due to the time spent by the algorithm for obtaining the value of syntactic or lexical similarity. This time this is much more important with the use of WORDNET than with another function of syntactic calculation of similarity like that of LEVENSHTEIN. Indeed, the use of the API WORDNET requires accesses expensive disc to seek synonymies.

\section{RESULTS}

When the mapping between the original ontologies LOM and MPEG-7, we found 3 cases:

\section{A. One LOM data corresponds to one MPEG-7 data :}

For example the MediaIdentificationType "EntityIdentifier" with item "identifier" in the category "general" LOM. The "EntityIdentifier" identifies uniquely the particular and unique multimedia content entity. For example, ISO's ISAN and the element "identifier " which is A globally unique label that identified the learning object.

Other mapping between the CreationType: "Title" with the element "title" of category "general" LOM. The first element describes one textual title of the multimedia content. Multiple titles are allowed. They may correspond to different types or to different languages.. And the second element is the Name Given to this learning object. The following table shows examples qulques direct mapping between MPEG-7 data and LOM data.

TABLE II.

EXEMPLE 1 OF TABLE OF CORRESPONDENCES BETWEEN LOM AND MPEG-7 ONTOLOGIES

\begin{tabular}{|l|l|}
\hline MPEG-7 element & $\begin{array}{l}\text { LOM Meta-data } \\
\text { element }\end{array}$ \\
\hline $\begin{array}{l}\text { MediaInfor- } \\
\text { mation.MediaIdentification.EntityIdentifier }\end{array}$ & general.identifier \\
\hline CreationInformation.Creation.Title & general.title \\
\hline CreationInformation.Classification.Language & general.language \\
\hline DescriptionMetadata.Version & lifeCycle.version \\
\hline MediaLocator.MediaURI & technical.location \\
\hline Segment.Relation & Relation \\
\hline
\end{tabular}

\section{B. One LOM data corresponds to multiple MPEG-7} data :

Given the richness of the MPEG-7, we see that several elements of the MPEG-7 were mapped in one LOM element, for example if we take the the "ClassificationType" which Describes the classification of the multimedia and especially the target of the multimedia content in terms of market classification, age and country as the "CreationCoordinates" aimed at describes the location and the date of creation of the multimedia content and other elements can be mapped to the element "coverage" of the category "general" which describes: The time, culture, geography or area to All which this learning object Applies.

TABLE III

EXEMPLE 2 OF TABLE OF CORRESPONDENCES BETWEEN LOM AND MPEG-7 ONTOLOGIES

\begin{tabular}{|l|l|}
\hline MPEG-7 element & $\begin{array}{l}\text { LOM Meta- } \\
\text { data element }\end{array}$ \\
\cline { 1 - 2 } CreationInformation.Classification.Target & $\begin{array}{l}\text { gen- } \\
\text { eral.coverage }\end{array}$ \\
\cline { 1 - 1 } CreationInformation.Creation.CreationCoordinates & $\begin{array}{l}\text { annota- } \\
\text { tion.entity }\end{array}$ \\
\cline { 1 - 1 } DescriptionMetadata.Creator.Agent.Person.Name & $\begin{array}{l}\text { DescriptionMetada- } \\
\text { ta.Comment.StructuredAnnotation.Who }\end{array}$ \\
\cline { 1 - 2 }
\end{tabular}

\section{No mapping :}

Note that limited elements of LOM cases remain without mapping, it is the element structure of the category "General" which is to Underlying students organizational structure of this learning object, Same thing for the property installationRemarks.

\section{CONCLUSION AND FURTHER WORK}

Finally, in this work; we try to find an appropriate solution to ensure syntactic interoperability of multimedia learning objects; by using the mapping between ontologies sources LOM and MPEG-7, however, the use of multimedia in this context requires the establishment of a semantic platform for learning in which the learner interacts with a device for inducing the discovery and construction of knowledge, and the integration of knowledge management and navigation " intelligent" in semantic learning platform.

Indeed, the next step is to measure the similarity of ontology mapping with other e-learning standards such as SCORM, IMS. even make this formal ontology operational and usable by an application.

\section{REFERENCES}

[1] IEEE Learning Technology Standards Committee (LTSC), "Draft Standard for Learning Object Metadata", Available at http://ltsc.ieee.org/wg12/files/LOM_1484_12_1_v1_Final_Draft.p df, 2002.

[2] A. Strijker, "Reuse of Learning Objects in Context : Human and Technical Aspects ", Thesis University of Twente, Enschede, 2004, pp. 1.

[3] J. Greenberg, "Metadata and the World Wide Web", Encyclopedia of library and information science, , Vol. 3, 2003, pp. 1876-1888.

[4] M. Nilsson, A. Naeve, E. Duval, P. Johnston, D. Massart, “ Harmonization of Metadata Standards “, Network of Excellence in Professional Learning PROLEARN, 2008, p. 4.

[5] José M. Martínez, "MPEG-7 overview", Available at http://www.chiariglione.org/mpeg/standards/mpeg-7, Palma de Mallorca, 2004 
[6] P. Salembier, "Introduction to MPEG 7: Multimedia Content Description Language", 2010, p.84.

[7] J. Hunter, "Adding Multimedia to the Semantic Web - Building an MPEG-7 Ontology", International Semantic Web Working Symposium (SWWS), Stanford, July 30 - August 1, 2001.

[8] Savithri Godugula, "Survey of Ontology mapping Techniques, Software Quality and Assurance", Aug 1,. 2008.

[9] P. Shvaiko, J. Euzenat, "Ontology matching: state of the art and future challenges", IEEE Transactions on Knowledge and Data Engineering, 2013. http://dx.doi.org/10.1109/TKDE.2011.253

[10] MILLER G. A., "WordNet : a Lexical Database for English ”, Communications of the ACM, vol. 38, n" 11, p. 39-41, November, 1995 .

\section{AUTHORS}

Jihad CHAKER is with Laboratory of Informatics, Research Operational and Statistic Applied (LIROSA) at Faculty of Sciences, Abdelmalek Essaadi University, Tetwan, Morocco. (e-mail: chaker.jihad@gmail.com).

Souhaib AAMMOU is with Laboratory of Informatics, Research Operational and Statistic Applied (LIROSA) at Faculty of Sciences, Abdelmalek Essaadi University, Tetwan, Morocco. (e-mail: Souhaib_111@yahoo.fr ).

Mohamed KHALDI is with Laboratory of Informatics, Research Operational and Statistic Applied (LIROSA) at Faculty of Sciences, Abdelmalek Essaadi University, Tetwan, Morocco. (e-mail: medkhaldi@yahoo.fr).

Submitted 11 june 2014. Published as resubmitted by the authors 19 August 2014 